\title{
A PROPERTY OF THE REAL NOT REGULAR FUNCTIONS $C^{\infty}$
}

\section{HANS HORNICH}

Let $f$ be $\in C^{\infty}$ on the interval $I=[0, \mathfrak{L}], \mathfrak{\&}>0$ and

$$
\lim \sup \left(\left|f^{(n)}(0)\right| / n !\right)^{1 / n}<+\infty .
$$

If $f$ is not regular for $x=0$, i.e. if $f$ cannot be expanded into a power series in $x$ on $[0, \delta]$ for any $\delta>0$, then there exists a $n(K)$ for every $K>0$ such that $f$ cannot be a solution of a linear differential equation on $I$

$$
y^{(n)}+\sum_{i=0}^{n-1} \sigma_{i} y^{(i)}=\varphi
$$

with any $\mathcal{L}$-integrable functions $\sigma_{i}$, $\varphi$ on $I$ with

$$
\left|\sigma_{i}(x)\right|<K, \quad|\varphi(x)|<K
$$

for $x \in I$ and $n>n(K)$.

Proof. If $f$ satisfies a differential equation (2) then

$$
\bar{f}(x)=f(x)-\sum_{i=0}^{n-1} f^{(i)}(0) x^{i} / i !=f(x)-P(x)
$$

satisfies a differential equation

$$
\bar{y}^{(n)}+\sum_{i=0}^{n-1} \sigma_{i} \bar{y}^{(i)}=\varphi-\sum_{i=0}^{n-1} \sigma_{i} P^{(i)}=\Phi
$$

with

$$
\bar{f}(0)=\bar{f}^{\prime}(0)=\cdots=\bar{f}^{(n-1)}(0)=0 .
$$

Now

$$
\sum_{i=0}^{n-1} \sigma_{i} P^{(i)}=\sum_{i=0}^{n-1} \sigma_{i} \sum_{j=i}^{n-1} f^{(j)}(0) x^{j-i} /(j-i) !
$$

and with

$$
\max _{i} \sup _{x \in I}\left|\sigma_{i}(x)\right|=S
$$

Presented to the Society, April 12, 1965; received by the editors March 12, 1965. 
we get the inequality

$$
\left|\sum_{i=0}^{n-1} \sigma_{i} P^{(i)}\right| \leqq S \sum_{i=0}^{n-1} \sum_{j=i}^{n-1}\left|f^{(j)}(0)\right| x^{j-i} /(j-i) ! .
$$

Because of (1) we have for an appropriate $A>1$

$$
\left|f^{(j)}(0)\right| \leqq A^{j j} \text { ! }
$$

so

$$
\begin{aligned}
\left|\sum_{i=0}^{n-1} \sigma_{i} P^{(i)}\right| & \leqq S \sum_{i=0}^{n-1} \sum_{j=i}^{n-1} A^{j j}(j-1) \cdots(j-i+1) x^{j-i} \\
& \leqq S A^{n-1} n^{2}(n-1) !
\end{aligned}
$$

for $x<1$.

We prove the following

Lemma. For the solution $\bar{y}$ of a differential equation

$$
\bar{y}^{(n)}+\sum_{i=0}^{n-1} \sigma_{i} \bar{y}^{(i)}=\bar{\phi}
$$

with

$$
\bar{y}(0)=\bar{y}^{\prime}(0)=\cdots=\bar{y}^{(n-1)}(0)=0
$$

the following inequality is valid:

$$
|\bar{y}(x)| \leqq \phi \& x^{n-1} /\left(1-\sum_{i=0}^{n-1} S_{i} \mathfrak{L}^{n-i} /(n-i) !\right)(n-1) !
$$

on $I=[0, \mathfrak{L}]$ with

$$
S_{i}=\sup _{x \in I}\left|\sigma_{i}(x)\right|, \quad \phi=\sup _{x \in I}|\bar{\phi}(x)|
$$

where $\mathfrak{L}$ is so small that

$$
\sum_{i=0}^{n-1} S_{i} \mathcal{L}^{n-i} /(n-i) !<1 .
$$

We put

$$
\sup _{x \in I}\left|\bar{y}^{(n-1)}(x)\right|=Y
$$

then it follows that

$$
\left|\bar{y}^{(i)}(x)\right| \leqq Y x^{n-1-i} /(n-1-i) !
$$


for $i=0, \cdots, n-1$.

Now from (10) we get

$$
\bar{y}^{(n-1)}(x)=\int_{0}^{x}\left[\delta(t)-\sum_{i=0}^{n-1} \sigma_{i}(t) \bar{y}^{(i)}(t)\right] d t
$$

and because of (14)

$$
\left|\bar{y}^{(n-1)}(x)\right| \leqq \phi x+\sum_{i=0}^{n-1} S_{i} Y x^{n-i} /(n-i) !
$$

and

$$
Y \leqq \phi \mathscr{L}+\sum_{i=0}^{n-1} S_{i} Y \mathfrak{L}^{n-i} /(n-i) !
$$

or

$$
\left|\bar{y}^{(n-1)}(x)\right| \leqq Y \leqq \phi \mathscr{L} /\left(1-\sum_{i=0}^{n-1} S_{i} \mathfrak{L}^{n-i} /(n-i) !\right)
$$

and finally by integration (12).

We apply this Lemma to (4); if $\mathscr{L}$ is supposed to be so small that

$$
\mathfrak{L}<1, A \mathfrak{L}<1 \text { and } S\left(e^{\mathfrak{L}}-1\right)<1 \text {, }
$$

then because of (9)

(19) $|\bar{y}(x)| \leqq\left[K+S n^{2} A^{n-1}(n-1) !\right] \& x^{n-1} /(n-1) !\left[1-\left(e^{\mathscr{L}}-1\right) S\right]$.

Now $f$ is not regular for $x=0$; therefore, for every natural number $m$ we have

$$
\sup _{x \in I}\left|f(x)-\sum_{i=0}^{m-1} f^{(i)}(0) x^{i} / i !\right|=h_{m}>0
$$

and

$$
\inf _{m} h_{m}=h>0 \text {. }
$$

There exists, for every $m$, an $x_{m}$ on $I$ with

$$
\left|f\left(x_{m}\right)-\sum_{i=0}^{m-1} f^{(i)}(0) x_{m}^{i} / i !\right| \geqq h .
$$

The right side of (19) becomes arbitrarily small for $n \rightarrow \infty$; there. fore, an $n(K)$ exists for a given $K$ such that

$$
|\bar{y}(x)|<h
$$


holds for $x \in I$, that is, for all functions $\sigma_{i}$, with

$$
S \leqq K, \quad|\varphi(x)|<K
$$

and $n>n(K)$. The inequalities (19) and (21) are both valid only if $m<n(K)$ holds in

$$
\bar{y}(x)=f(x)-\sum_{i=0}^{m-1} f^{(i)}(0) x^{i} / i !
$$

We add some consequences. Let $f$ be $\in C^{\infty}$ on $I=[0, \&]$. If

[i] for every point $x \in I$ there is

$$
\lim \sup \left(\left|f^{(n)}(x)\right| / n !\right)^{1 / n}<+\infty
$$

and

[ii] if there exists a sequence of linear differential equations of increasing order with uniformly bounded coefficients such that $f$ satisfies every differential equation of this sequence, then $f$ is regular in every point of $I$.

This is an immediate consequence of our theorem.

The validity of (23) in every point of $I$ is a necessary condition for the regularity of $f$.

If $f$ satisfies a linear differential equation with uniform bounded coefficients for every order $n>n_{0}$, then (23) is valid for every point of $I$ and $f$ is regular on $I$.

It is necessary to give the proof for the first part only; if (23) is not valid for a point $x_{0}$ on $I$, then there exists a subsequence $\left\{n_{K}\right\}$ of natural numbers such that $\left|f^{\left(n_{K}\right)}\left(x_{0}\right)\right|$ are increasing in such a way that the equation (2) cannot be true for the point $x_{0}$ for sufficiently large $n_{K}$.

Catholic University of America 